nommen sind nur die ungedruckten Manuscripte, das bare Geld und einige Gegenstände, die dem Verewigten aus den höchsten Kreisen schenkungsweise überkommen sind. Ueber diese hat er miltelst kleiner Zettel, zu Gunsten befreundeter Personen besondere Dispositionen getroffen. Unter den ungedruckten Manuscripten befindet sich ein vollendetes geographisches Werk von bis jetzt nicht existent gewesenem Umfange, das natürlich, wie alle anderen derarligen Werke, Eigenthum der Erben des Autors wird. An baaren Geldern und geldwerthen Papieren sind nur 400 und einige Thaler vorhanden gewesen. $V_{0}$ diesen hatte der Verstorbene, der eine kleine Pension rom Staate bezog, bereits bei Lebzeiten 400 Thaler seinem alten Diener mit der schriftlichen Erıächtigung, damit die Kosten seines Begräbnisses zu bestreiten, überlassen. Welchen Werth der grosse Mann auf persönliche Auszeichnungen im Leben gelegt hat, darüber liefert die Thatsache ein charakteristisches Merkmal, dass die ihm aus aller Herren Länder zugestrōmten Orden, wie sie in solcher Zahl und von solcher Bedeutung wohl noch nie auf der Brust eines einfachen Gelehrten vereinigt worden, durchaus ungeordnet in einem ziemlich vernachlässigten Schrank aufbewahrt wurden. Eine Bestimmung, in welcher W eise die irdischen Reste des unsterblichen Mannes zur Gruft bestattet werden sollen, hat der Verewigte, der mit klarer Erkenntniss seine nahe Auflösung vorhergesehen und deshalb sehr specielle Dispositionen, soweit sie andere Personen betreffen, erlassen hat, nicht errichtet. Als gesetzliche Erben sind die Kinder und Enkel Wilhelm v. Humboldt's - Bruders des Verewigten - legitimirt.

\title{
Notizen über rexbus.
}

\section{Von Joh. Bayer.}

Schon viele Botaniker haben es als eine verlorene Abmühung erkannt, ein Rubus-Exemplar, wie solche gewöhnlich in den Herbarien liegen, oder von Excursionen nach Hause gebracht werden, nach der Beschreibung eines Autors oder nach einer Abbildung mit Sicherheit bestimmen zu wollen. Hieraus folgt aber noch nicht die völlige Anarchie in den Formen dieser Pflanze, oder die Unmöglichkeit einer Gruppirung derselben; sondern das folgt; dass ein anderer Weg eingeschlagen werden müsse, als jener, auf welchem man sich mit konstanter begrenzten Formen zurechtfindet.

Wenn es wahr ist, dass der Systematiker den wissenschaftlichen Bolanikern, d. i. den Morphologen, Organographen und Thysiologen nur als Handlanger dient, so ist es doch auch eben so wahr, dass beide, um sich gegenseitig versländigen zu können, dem Materiale, dessen sie sich bei ihren Arbeiten bedienen, einen Namen geben müssen, um den Babel endlich mit einander aufzubauen.

In Beziehung auf die Brombeersträucher war das Besireben der Systematiker seither, wie ich glaube, eifriger als das Studium 
der wissenschaftlichen Botaniker, wodurch die ersteren einer ausgiehigen Unterstützung und Leitung entbehrten. Jedenfalls feblt bei der selbstständigen Aufstellung einer jeden einzelnen Form ohne das Zusammenwirken der genannten Wissenschaften ein grundfestes Princip, und sie muss, von allen Seiten gerüttelt, bald wieder zusammen fallen.

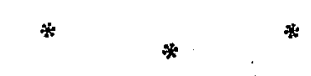

Vor Allem ist es nothwendig, das Auge an die Eindrïcke der lebendig en Formen zu gewöhnen, und das allgemeine Bild einer Gruppe von Formen festzuhalten, um nach und nach einzelne Merkmale besonders aufzufassen und zu einem charakteristischen Bilde vereinen $\mathrm{zu}$ können.

Die Schösslinge mancher Formen steigen, von dem benachbarten Gesträuche unterstiitzt, und dem Lichte folgend, oft zu einer bedeutenden Höhe, biegen sich dann in weiten Bogen abwärts, und wachsen so mit ihren Spitzen der Erde zu, wo sie einwurzeln und wie $\mathbf{A b -}$ senker neue Individuen bilden. Je günsliger der Spätsommer dem Gedeihen einer solchen Pflanze ist, desto höher steigł der Schössling; desto weiter ist aber auch der Weg, auf welchem er noch vor dem Eintritte des Winters den Boden wieder erreichen soll. Daher beeilt sich derselbe um so mehr, diesen in der kürzesten Linie, und mit immer mehr verlängerten Internodien zurückzulegen, je rauher die HerbstTemperatur wird; so dass die absteigende Hälfte des Schösslings oft in halb so kurzer Zeit gebildet wird, als die aufsteigende. Diese Verhältnisse sind meines Wissens in die phänologischen Beobachtungen noch nicht aufgenommen worden, wo sie, wie manche andere, einen Platz finden könnten, und wozu sich einige Formen besonders eignen dürften.

An solchen Schösslingen stehen die geraden Stacheln von dessen Basis bis auf den ScheiteI des Bogens senkrechl an denselben (horizontal abstehend); an jenem Theile aber, welcher sich von da zur Erde senkt, richten sie sich, so wie die Blätter mit ihren Stielen gegen das Licht a ufwärts, d. h. von der Spitze des Schösslings z u r ü ck, und zwar um so mehr, je senkrechter dieser Theil sich niederlässt, so wie dieses an den Bogen der Rosenschösslinge eben auch der Fall ist. Es ist daher überflüssig, in den Diagnosen den Terminus aufzunehmen: „Stacheln am oberen Theile des Schösslings z u r ü ckgerichtet." Doch liegt die Anlage zu dieser Rückrichtung schon in der Natur des Schösslings, indem die Stacheln der oberen Hälfte jener Schösslinge, welche keinen Stülzpunkt finden, und daher liegend fortwachsen, mehr weniger auch zurückgerichtet erscheinen. Dieser Umstand kann an einem einzelnen Herbarstücke nicht beobachtet werden, wenn es nicht der Bogen selbst ist, an welchem beide Richtungen vorkommen.

Die Schösslinge senken ihre Spitzen oft in den benachbarten Strauch einer andern Form, wo sie dann Wurzeln schlagen, und im nächsten Jahre ein neues Individuum bilden. Hiedurch entsteht ein 
Gemisch von mehreren Formen in Einer Hecke, in welcher in verschiedenen Jahren, ja in verschiedenen Jahreszeiten, bald die Eine bald die Andere das Uebergewicht erringt, welches gewöhnlich von den individuellen Entwicklungsphasen abhängt. Es lässı sich begreifen, welche Confusionen bei einem oberflächlichen Sammeln dadurch veranlasst werden. *)

Die Ruckseite der untersten Blälter eines Schösslings ist oft grün, die der höheren dem Lichte näher kommenden aber immer mehr grau filzig. Dieser Umstand kann, ausser der Einwirkung des Lichtes, oft der Vergrösserung der Blattfläche im Schatten, und der dadurch bedingten Auseinandertretung der Haare zugeschrieben werden. Auch hierauf wird beim Einsammeln selten Rücksicht genommen.

Die Schösslinge mancher Formen haben constant nur 3zählige, oder n u r 5-zählige, und wieder andere conslant 3- u n d 5-zählige, oder 3- o der 4- und 5-zählige Blättchen. Die Regel, dass einem Herbar-Exemplar auch ein Stück des Schösslings mit einem Blatte beizulegen sei, ist daher eben auch nicht genügend, weil das im Freien oft sicher bezeichnende Kennzeichen der 3- o de r 5-zähligen oder der 3- oder 4- und 5-zähligen Blättchen nicht ersehen werden kann, wodurch dann die Ansichten über die Veränderlichkeit der Formen noch mehr auseinander gebracht werden.

Aus diesen wenigen Beobachtungen ist zu ersehen, dass die Bestimmung der meisten Herbar - Exemplare derzeit fast unmöglich ist, und dass das oft ausgesprochene Urtheil über die ausserordent lichen Abweichungen der Formen dieses Genus einige Beschränkungen zulässl.

Ich will nur noch aus den nächsten Umgebungen Wiens beispielweise einige Formen bezeichnen, welche sich unter der grossen Anzahl anderer ohne Schwierigkeiten erkennen lassen. Ich sehe dabei von einer vollständigen Diagnose $a b$, und führe nur jene Merkmale an, welche im F r ei e n zur Unterscheidung genügen, ohne die Abänderungen der einzelnen Theile zu berühren; denn wer einmal eine Hauptform genau erkannt hat, wird auch jene leichter unterbringen.

Rub us fastigiatus W.N. - Kelchabschnitte braung r ü $\mathrm{n}$ mit weisser Einfassung, $\mathrm{g}$ a $\mathrm{nz} \mathrm{k}$ a h l; Blüthen gross, in einer fast einfachen, fl a c h g i p f el ig e n Traube, rosa; Blättchen beiderseits grün. - Dieser äusserst schöne Strauch, mit seinen blüthenreichen Bogen, ist um Wien ziemlich selten, z. B. in der Schlucht hinter dem Dornbacher Parke, rechts vom Wege zur Rohrerhülte,

*) Man kann z. B. auf dem Gallizin Hecken beobachten, in welchen im Sommer nur $R$. eillicaulis zu sehen ist; im Herbste aber scheinen sie gänzlich in $R$. macroacanthos umgewandelt. Nach genauen Beobachtungen findet man, dass der $R$. villicaulizi vorerst durch seine Stärke das Uebergewicht gewinnt; da aber dessen Blätler früher welk werden, und früher abfallen, als jene des $R$. macroacanthos, welche auch noch im Wiuter frisch bleiben, so maskirt dieser den ersten bald vollständig. 
wo er gegen Ende Juni bluht. - Die verwandten Formen, mit welchen er rücksichtlich des Kelches verwechselt werden könnte, sind mir in der Nühe noch nicht vorgekommen.

R. thy rsoideus Wim m., R. fricticusus W.N. - Kelch graufilzig, Blüthen in (meist grossen) Sträussen, rosa; Blältchen i n die Länge gezogen, vom oberen Drittel gegendie $B$ asis verschmälert, die untersten unterseits grün, die nach oben folgenden mehr und mehr graufilzig, Mittelrippe an der Unterfläche stark hervorgehoben, meist fl a c it mit zwei Längsfurchen, gelbJich oder von der. Farbe des Schösslings. - Ain ausgezeichnetsten an etwas schattigen Orten, in Holzschlägen ziemlich häufig. - Blüht anfangs Juli. - Nach den Blättern könnte er mit $R$. tomentosus verwechselt werden; doch sind beide durch den Geruch der Bläthen, die Form der Blüthenblälter, die Griffel und Stacheln verschieden. - Von den übrigen ähnlichen unterscheidet er sich durch den nach oben ganz kahlen (nur böchst selten etwas zerstreut-flaumigen) Schössling und eben solche Stacheln, von welchen die Basis der stärksten ein gleichseitiges Dreieck bildet.

R. tomentosus Borkh. - Ist schon an dem Honiggeruche der Blüthen (wie Host richtig sagt) allein zu erkennen; ausser diesem an den la $\mathrm{l} g \mathrm{e}, \mathrm{k}$ e $\mathrm{i}$ l fö $\mathrm{r} \mathrm{m}$ ig e $\mathrm{n}$, weissen Blüthenblätlern, und an den feinen, ganz strohgelben Stacheln auf niedriger Basis, welche oft nur einen langen, wenig erhabenen Strich bildet. - - Um Wien häufig, z. B. bei Mauer in dem Föhrenwäldchen gegen den kais. Thiergarten in ausgebreiteten Gruppen, welche sich schon von fern durch die sleif-aufrecht stehenden Rispen und ihren Geruch bemerkbar machen; blüht yom Anfang Juni fast durch den ganzen Sommer. Wegen einer Verwechslung mit R.thyrsoideus siehe jenen. Mit irgend einem $R$. discolor, oder gar $R$. fructicosus $\mathbf{L}$. (R. plicalus W.N.) hat er kaum eine Aehnlichkeit.

R. macroacanthos W. N. - Schössling fast kahl, Stacheln behaart; Blättchen unterseils weissfilzig, zu $\mathbf{3}$ u n d zu $\mathbf{5}$, oder zu 3 oder 4 und 5 an demsel be n Schösslinge; bei den fünfzähligen ist das unterste, am Stiele des zweiten, stehende Blättchen auffallend länger gestielt, als bei den verwandten Formen. - Dieser Strauch scheint anderwärts weniger häufig zu sein, als um Wien, wo er, z. B. um Dornbach und auf dem Gallizin grosse Hecken bildet, und Anfangs Juli rosenrolh blüht. - Durch die Zahl der Blättchen und durch den dünneren, oft peitschenförmigen Schössling unterscheidet er sich leicht von den ähnlichen, mit welchen er oft untermischt wächst. - Der diesem am meisten ähnliche, und mit ihm vorkommende ist

R. $p u$ bes cens W. N., welcher aber nur 5-zählige Blättchen hat, von welchen jene Eines gemeinschaftlichen Blattstieles in i h r er Form alle einander gleich, und nur in der Grösse verschieden sind.

R. py g n a e us W.N. - Schössling niedergestreckt, drüsen-

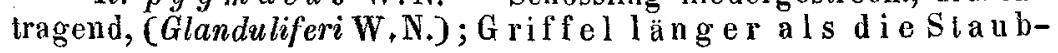


g e fä s s e, grün; dadurch von allen ähnlichen verschieden. - Blüht weisslich im Juli. - Um Wien nicht häufig, z. B. auf dem Rosskopf in Holzschlägen.

Von R. hirtus W.K. ist eine Form (Var. giganteus Ne uma $n \mathrm{n}$ in Herb.) ausgezeichnet: Der Blülhenstengel erhebt sich a u $\mathrm{s}$ dem Moose und a us alten Baumblättern bis gegen $3^{\prime}$ hoch, wenn er eine Stütze findet, sonst liegt er auf der Erde, und ist vom Grunde bis an die Rispe in seinen Blattwinkeln mil vielen rispenblüthigen Aestchen $z w e$ iseitswendig besetzt; den Umriss des ganzen grossen Blüthenstengels mit seinen Blättern und Seitenrispen könnte man lanzettförmig nennen. - Er findet sich immer nur einzeln in Holzschlägen oder an Waldrändern und fällt besonders durch die Menge seiner Früchle auf, welche ein einziger solcher Stengel liefert. - Blüht weisslich im Juli.

R. dum et or u $m$ W. N., R. nemorosus H y n e. - Bei den gewöhnlichen Formen ist das Endblätıchen der 5-zähligen Blätter über das gewöhnliche Verhältniss gross, breit, rundlich, die untersten zwei Blättchen sind klein, sitzend; die Griffel röthlich, Früchte schwarz (nicht blau bereift). - Je nach den verschiedenen Standorten in sehr vielen Abänderungen häufig. - Blüht roth oder weiss schon Anfangs Juni.

Zunächst können dessen Formen mit $\boldsymbol{3}$ Blättchen mit $\boldsymbol{R}$. caesius verwechselt werden; sie unterscheiden sich aber durch die Farbe der Griffel, die nicht blau bereiften Früchte, und dass die meisten Kelchzipfel von der Frucht abstehend, nicht aber a lle anliege n d sind.

R. caesius L. - An den 3-zähligen Blättchen, den pergamentartigen Kelchzipfeln, welche alle der Frucht anli e ge $n$, an den g $\mathbf{r}$ ü $\mathrm{n}$ Griffeln und den bla u bereiften Früchten, mit wenigen grossen Theilfrüchten, leicht zu erkennen. In verschiedenen Formen fast überall, und auch fast durch den ganzen Sommer (gross, weiss) blühend.

R. I da eus L. - Untere Blälter gefiedert; Blüthenblätter a ufrecht; Früchte roth, fla u m ig. Allgemein bekannt.

R. saxatilis L. - Krauta rtig; Blättchen alle 3-zählig; Früchte mit nur 2-4 Theilfrüchtchen, roth. - Auf steinigen, gebirgigen Waldplälzen. - Blüht meist im Mai und Juni. ${ }^{*}$ )

Die übrigen um Wien wachsenden Formen lassen sich mit wenigen Worten nicht so leicht kennzeichnen; ist man aber nur erst mit einigen genau vertraut, so wird es dann leichter, die Zahl der bekannten zu vermehren.

$\mathrm{W}$ i e n, am 9. April 1859.

*) Der Herr Verfasser hat für die Besitzer dieser Zeitschrift von den hier angeführten Brombeeren eine Anzahl Exemplare in mehreren Formen zur Disposition gestellt, welche daher vou der Redaction auf Verlangen unentgeltlich bezogen werden könner. Die Redaction. 